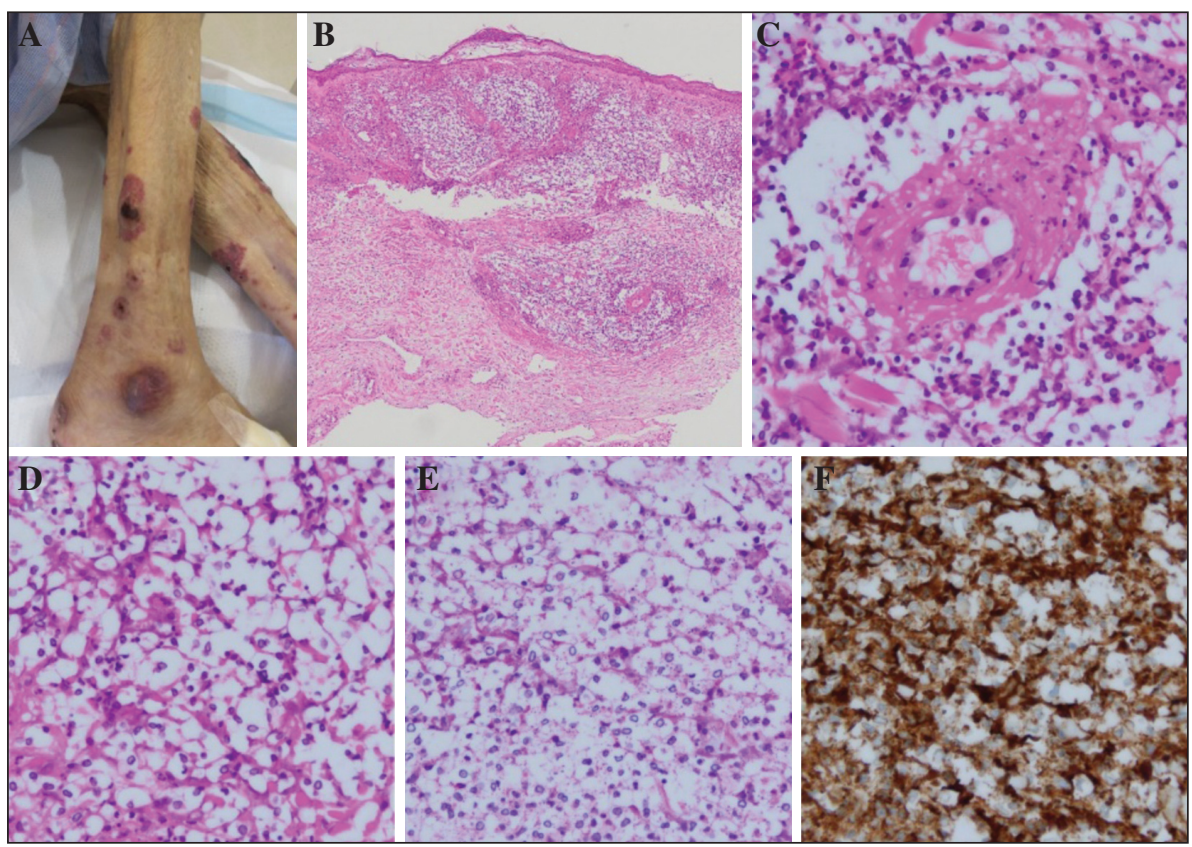

Figure 1. A) Skin lesions on the right lower leg showing purpuric papules and plaques with crusts. B, C) Leukocytoclastic vasculitis of the dermal vessels with infiltration of lymphocytes and neutrophils (haematoxylin and eosin stain; original magnification: $\times 40[\mathbf{B}], \times 400[\mathbf{C}])$. D) Numerous yeast-like pale basophilic bodies surrounded by capsule-like vacuolated spaces (original magnification: $\times 400$ ). E) Negative periodic acid-Schiff staining of the basophilic bodies (original magnification: $\times 400$ ). F) Diffuse immunostaining for myeloperoxidase (original magnification: $\times 400$ ).

"cryptococcoid Sweet syndrome" has been proposed [5]. In addition, leukocytoclastic vasculitis with cryptococcus-like changes has been described, and these patients had positive serum MPO-ANCA [4]. To the best of our knowledge, this is the first report of RV mimicking cryptococcal infection. Although the aetiology of this morphological change remains unclear, it is speculated that it represents degenerative changes in neutrophils. A previous report using transmission electron microscopy demonstrated that the basophilic bodies and vacuolated spaces were consistent with remnants of degenerating human cells [3]. Immunostaining for MPO was also positive, further supporting the neutrophilic origin of the cells. The treatment for RV includes systemic corticosteroids, while for cryptococcosis, it is important to consider reducing immunosuppression and administering systemic antifungal agents. Therefore, recognition of this histological change is essential to avoid misdiagnosis and inappropriate therapy in patients with RA presenting with cutaneous lesions.

Disclosures. Financial support: none. Conflicts of interest: none.

\footnotetext{
${ }^{1}$ Department of Dermatology, Japanese Red Cross Kitami Hospital, Kitami, Japan

${ }^{2}$ Department of Internal Medicine, Japanese Red Cross Kitami

Hospital, Kitami, Japan

${ }^{3}$ Department of Dermatology,

Asahikawa Medical University,

Asahikawa, Japan

<iinuma@asahikawa-med.ac.jp>
}

1. Kishore S, Maher L, Majithia V. Rheumatoid Vasculitis: A Diminishing Yet Devastating Menace. Curr Rheumatol Rep 2017; 19:39.

2. Noguchi $H$, Matsumoto $T$, Kimura $U$, Hiruma $M$, Kusuhara $M$, Ihn H. Cutaneous Cryptococcosis. Med Mycol J 2019; 60: 101-7.

3. Ko JS, Fernandez AP, Anderson KA, et al. Morphologic mimickers of Cryptococcus occurring within inflammatory infiltrates in the setting of neutrophilic dermatitis: a series of three cases highlighting clinical dilemmas associated with a novel histopathologic pitfall. J Cutan Pathol 2013; 40: 38-45

4. Fresco A, Wang J, Krausz A, et al. Cryptococcus-like changes in the setting of vasculitis. J Cutan Pathol 2019; 46: 143-7.

5. Wilson J, Gleghorn K, Kelly B. Cryptococcoid Sweet's syndrome: Two reports of Sweet's syndrome mimicking cutaneous cryptococcosis. J Cutan Pathol 2017; 44: 413-9.

doi:10.1684/ejd.2021.4055

\section{COVID-19 hand hygiene measures for health care workers}

As the COVID-19 pandemic is still developing, it is becoming more and more evident that currently recommended hand hygiene measures to limit the spread of the virus should be respected whenever and wherever possible. Different health authorities worldwide recommend frequent hand washing with soap or similar detergents and water, the use of alcohol-based hand sanitizers (ABHS) and wearing of protective gloves and face masks [1-6]. Even though these hygiene measures are recognised as being effective, intensive hand cleansing and disinfection, as well as the use 
of protective gloves, have been reported to cause adverse reactions on hands more and more frequently $[4,7,8]$. Therefore, taking care of the hands is of the greatest importance for all health care workers (HCW).

We performed a literature search in Pubmed and on health authority websites for the most recent reports on hand skin damage following the frequent use of water and detergents or ABHS or from wearing protective gloves before and after the onset of the COVID-19 pandemic, hand skin damage related to COVID-19 pathophysiology, as well as recommendations regarding prevention and care (figure 1).
The following key words were used: "hand" "+hygiene", "+skin damage", "+dermatitis", "+eczema", "+dishydrosis", "+COVID-19", and "skin lesions".

As this review focuses on the consequences of frequent hand hygiene measures during the COVID-19 pandemic in $\mathrm{HCW}$, as well as international recommendations for hand skin care, the different features of contact dermatitis, frequently observed on hands due to external irritants or antigens leading to its onset, were deliberately not covered. During the COVID-19 pandemic, in China, hand hygiene measures resulted in a $100 \%$ increase in skin lesions when

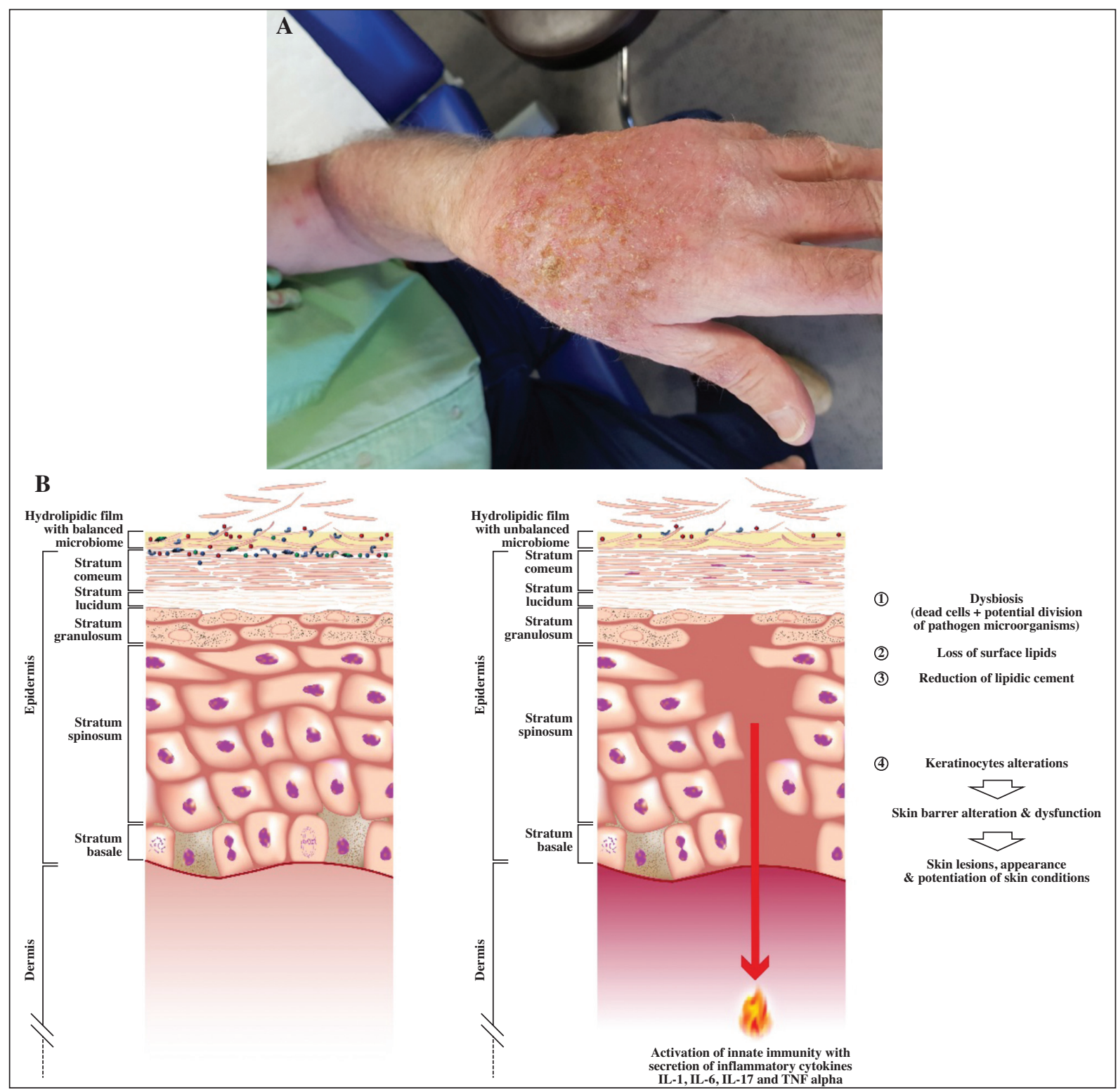

Figure 1. Skin damage caused by frequent hand hygiene. A) Contact dermatitis of a health care worker caused by frequent hand hygiene. B) Schematic diagram of the pathophysiology of skin lesions caused by frequent hand washing and use of ABSH. Lesions caused by frequent hand washing and the use of ABHS mainly affect the epidermis. Frequent hand washing using hot water and soaps with a high $\mathrm{pH}$ or hand sanitizers, or frequently using ABHS, alters the skin barrier and skin surface film, resulting in dysbiosis and activation of skin innate immunity and inflammation. Moreover, the corneocyte layer and the natural moisturizing factors of the granular layers are altered and the enzymatic activity in the intercellular space is increased, resulting in greater transepidermal water loss and an alteration of the composition of the hydrolipidic layer on the skin surface. 
hygiene routines were performed more than 10 times/day, and $74.5 \%$ of HCW reported self-perceived hand skin damage $[4,7]$. UK data showed that the prevalence of irritant hand dermatitis in hospitals caused by an increased frequency in hand washing and the use of ABHS was $62.5 \%$ [9]. In Germany, $90.4 \%$ of $\mathrm{HCW}$ were reported to have symptoms associated with acute hand dermatitis [10].

To date, more than 10 countries worldwide have published guidelines and recommendations for the prevention and treatment of hand skin lesions caused by frequent hand hygiene. Guidelines and recommendations are summarised hereafter.

Overall, HCW should avoid harsh washing with overly hot water, and prioritise the use of hypoallergenic foam cleansers or syndets with a $\mathrm{pH}$ of 5.5, which do not contain fragrances or antibacterial agents. After washing, hands should be gently dried without any friction or pressure, preferably using paper towels. ABHS with an alcohol concentration of at least $60 \%$ should be favoured and intense friction and pressure should be avoided. The use of gloves should preferably be limited to specific tasks, such as frequent contact with water or liquids, and cotton gloves should be used in addition, if necessary. Once protective gloves are taken off, hands should be washed and dried gently, as described previously.

Specific dermocosmetics are internationally recommended to restore, as quickly as possible, or prevent alteration of the skin barrier, the hydrolipidic layer and the cutaneous microbiome, due to frequent hand washing, the use of ABHS, and wearing of protective gloves. These prevent and reduce skin lesions caused by frequent hand hygiene measures, especially during the COVID-19 pandemic. Cleansers, moisturizers and repair creams provide maximum care of skin lesions on hands. To restore the natural skin barrier, fragrance-free, water-rich emollients or moisturizers with a $\mathrm{pH}$ of 5.5 should be used preferentially and should be applied as often as possible during work time and whenever hands are cleansed. Enriched formulations, such as specifically developed repairing balms which help to restore the microbiome and the natural skin barrier, may be used at home. To manage more severe forms, allergies, or secondary infections, treatment with topical corticosteroids in addition to emollients or moisturizers may be initiated, and patients with allergic reactions may be prescribed antihistamines. Secondary infections such as fungal and bacterial infections may be treated with antifungals or antibiotics.

Moreover, standardised educational programs may help to create awareness about skin damage due to hand hygiene.

Acknowledgements and disclosures. Acknowledgements: Brigitte Dréno acknowledges the writing support of Karl Patrick Göritz, SMWS-France. Conflicts of interest: none. Funding: this publication was supported by NAOS, Lyon France.

Department of

dermato-cancerology, CHU Nantes,

CIC 1413, CRCINA, INSERM,

University of Nantes, Nantes France

<brigitte.dreno@wanadoo.fr>
1. SFD. Société Française de Dermatologie. Mesures COVID-19: Prenez soin de vos mains. 2020 [Available from: https://evenementssfd.fr/?mailpoet_router\&endpoint=view_in_browser\&action=view\& data=WzE5NiwiNTQ5YTQzYTI4MTYzliwwLDAsMTQyLDFd. last accessed 25 January 2021.

2. CDC. Centers for Disease Control and Prevention. Prevention of Coronavirus Disease 2019 (COVID-19). 2020 [Available from: https://www.cdc.gov/coronavirus/2019-ncov/prepare/ prevention. html. last accessed 25 January 2021.

3. WHO. World Health Organisation: Wash and COVID-19 2020 [Available from: https://www.who.int/water_sanitation_health/newsevents/wash-and-covid-19/en/. last accessed 25 January 2021.

4. Yan Y, Chen H, Chen L, Cheng B, Diao P, Dong L, et al. Consensus of Chinese experts on protection of skin and mucous membrane barrier for health-care workers fighting against coronavirus disease 2019. Dermatol Ther 2020: e13310.

5. Robert Koch Institut. Empfehlungen des RKI zu Hygienemaßnahmen im Rahmen der Behandlung und Pflege von Patienten mit einer Infektion durch SARS-CoV-2. Stand 8.12.2020 [Available from: https://www.rki.de/DE/Content/InfAZ/N/Neuartiges_Coronavirus/ Hygiene.html. last accessed 25 January 2021.

6. Public Health. Guidance. COVID-19 personal protective equipment (PPE) 2020 [Available from: https://www.gov.uk/government/ publications/wuhan-novel-coronavirus-infection-prevention-and-

control/covid-19-personal-protective-equipment-ppe. last accessed 25 January 2021

7. Lan J, Song Z, Miao X, Li H, Li Y, Dong L, et al. Skin damage among health care workers managing coronavirus disease-2019. J Am Acad Dermatol 2020; 82(5): 1215-6.

8. Greveling K, Kunkeler ACM. Hand eczema pandemic caused by severe acute respiratory syndrome coronavirus 2 hygiene measures: the set-up of a hand eczema helpline for hospital personnel. J Eur Acad Dermatol Venereol 2020; 34(10): e556-7

9. Hadjieconomou S, Hughes J, Kamath S. Occupational skin disease during the COVID-19 pandemic, as captured in a Dermatology staff clinic in the United Kingdom. J Eur Acad Dermatol Venereol 2020; 34(11): e670-1.

10. Guertler A, Moellhoff N, Schenck TL, Hagen CS, Kendziora B, Giunta RE, et al. Onset of occupational hand eczema among healthcare workers during the SARS-CoV-2 pandemic: Comparing a single surgical site with a COVID-19 intensive care unit. Contact Dermatitis 2020; 83(2): 108-14

doi: $10.1684 / \mathrm{ejd} .2021 .4064$

\section{Mild hypertrichosis in both upper arms around dupilumab injection sites}

Dupilumab is an anti-interleukin (IL)-4 and IL-13 receptor $\alpha$ antibody, which inhibits T helper 2 (Th2) reactions and is approved for the treatment of severe refractory atopic dermatitis. Dupilumab has also been shown to induce hair regrowth in patients with alopecia areata associated with atopic dermatitis [1-3], however, the mechanisms of hair regrowth in response to dupilumab in cases of alopecia areata still remain unclear. Recently, we investigated a patient with atopic dermatitis who developed hypertrichosis in both upper arms around the injection sites of dupilumab. A 49-year-old man was referred to our hospital for generalized erythema. He had suffered from atopic dermatitis from childhood. He had been treated with oral levocetirizine ( $5 \mathrm{mg}$ /day) and topical delgocitinib ointment, but the erythema was unresponsive. Physical examination revealed 